\title{
Editorial
}

\section{Covert surveillance by doctors for life-threatening Munchausen's syndrome by proxy}

\author{
Raanan Gillon Imperial College Health Service and St Mary's Hospital Medical School, London University
}

An exchange in recent issues of the journal raises important questions about the ethics of covert video surveillance by doctors when they suspect lifethreatening Munchausen's syndrome by proxy. (This is the condition in which psychologically disturbed parents - usually mothers - inflict harm upon their children, especially and most dangerously, suffocation, in order, it is hypothesized, to gain medical attention for their children and thus, vicariously, for themselves.)

First it is important to distinguish two linked but clearly different moral issues. The paper by Dr Donald Evans (1) primarily concerned whether or not covert video surveillance and associated investigations in such cases should still be regarded as research, and therefore still require assessment by Research Ethics Committees (RECs) before implementation. However, the response to his paper from Professor David Southall and Dr Martin Samuels (2) primarily concerned the moral justification of covert video surveillance by medical and nursing staff, in certain sorts of extreme cases.

So far as submission to RECs is concerned, it seems important to start by reiterating what was not clear in the paper by Dr Evans but which was made explicit in the response by Professor Southall and Dr Samuels - namely that Professor Southall and Dr Samuels sent the protocol for their proposed covert surveillance to the local REC. What, however, Dr Evans was pointing out was that they had not sent it for review as a research proposal. This was true - for they believed and argued that it no longer constituted research - the technique had been, in their opinion, sufficiently researched previously (with REC approval) and was now an established method of clinical practice in the very rare circumstances for which it was considered appropriate.

Nonetheless they had sent their protocol to the local REC, arguing their case that it should not need assessment by that body since it no longer constituted research but was now established clinical practice. In doing so they were obviously inviting the REC to agree with them that the protocol was not something with which the REC should concern themselves - equally obviously they were giving the
REC an opportunity to disagree with their reasoning and require that the protocol should be assessed as a clinical research project.

Once the REC had been given the protocol, responsibility for deciding whether or not it should be assessed as clinical research had properly passed to a body set up to make such decisions, namely the REC. Clearly in this particular case the local REC agreed that the proposed protocol did not constitute clinical research and therefore did not fall within their remit. Instead, they apparently recommended that the protocol should be reviewed by a hospital ethics committee. Once such a committee had been established - for they are rare in the United Kingdom - 'our piotocol, established by excellent inter-agency collaboration between ourselves and Staffordshire Police, was presented, reviewed and approved' (2).

Readers will decide for themselves whether or not they accept Dr Evans's arguments that the work did constitute research and therefore should have been reviewed by a REC. What seems clear is that these arguments were not explicitly addressed, let alone refuted, by Professor Southall and Dr Samuels. Instead, the thrust of their paper was first to make clear that they had submitted their protocol to both a REC and a clinical practice hospital ethics committee and second to make clear that they would have preferred the police to have taken responsibility for the covert video surveillance, as the Metropolitan Police had done previously when similar work had been carried out in London.

Their third thrust was to argue against the claim that, in all cases, children can be adequately protected by relying on co-operation with the parent or parents, through open discussion with them of the suspected diagnosis, followed if necessary by a period of separation to see if the problem ceases, and to see if psychotherapeutic intervention with the suspected parent confirms and perhaps remedies the suspected diagnosis. And they poured scorn on the idea that overt video surveillance of mother and child is likely to succeed: 'Common sense dictates that this is inappropriate. If the mother knows her child is being videoed, how likely is she to suffocate him?' (2). Thus the third thrust of their response to Dr Evans is not a reply to any of his specific 
arguments for why their work should have been reviewed by a REC, but rather to argue why they consider it to be morally justified clinical intervention.

It is in this context that the most poignant moral dilemma seems to arise. On the one hand the moral tradition of medicine is to trust the patient and in any case not to impose treatment or any other medical intervention in the absence of consent, whether explicit, implicit or given by an acceptable proxy. Quite clearly none of these three types of consent is given for covert video surveillance in suspected lifethreatening Munchausen syndrome by proxy. Instead doctors and nurses become involved in deliberate deception of a child's parent, usually the mother; deliberate and systematic covert invasion of her privacy, to the extent of electronic spying on her in her bedroom, a paradigm symbol of privacy in our society; in short, deliberate adoption of modes of investigation that even when carried out by police and security services leave room for major moral unease about the associated infringement of civil rights and liberties. How much more disturbing are such investigations when carried out by doctors and nurses, professionals for whom the promise and creation of trust for both patients and the general public is a sine qua non of their professional functioning.

On the other hand, children, not their parents, are the primary patients for paediatricians, and they know from experience and the literature that a very small but tragic proportion of those patients are abused, sometimes to the extent of torment, sometimes even to death, by one or both parents, the very people entrusted with their care and protection. Thus while paediatricians acknowledge and respect the crucial role of the vast majority of parents as bona fide representatives, advocates, protectors and champions for their children, they must also develop strategies for recognising and then responding to the tiny minority of abusing parents, in the interests of protecting those unprotected offspring.

While it is true that in the vast majority of cases doctors should assume that it is the parents who are the representatives of the interests of their young children, and respect the autonomous views of the parents concerning medical proposals for interventions, and not impose any intervention against the will, or without the knowledge, of the parent acting as the child's proper proxy, sometimes these assumptions are unjustified. When there is sound evidence that the parent is acting against the interests of the child, and endangering the child, the customary right of the parent to make decisions on behalf of the child may be forfeited. It is upon this basis that the doctor is justified in informing the social services, and so directly or indirectly the police, when he or she suspects abuse. It is upon this basis that interdisciplinary case conferences are called to assess the evidence of abuse and decide upon the appropriate course of action to recommend to the courts.
In some cases, including some life-threatening versions of Munchausen syndrome by proxy, the diagnosis of the child's life-threatening disorder is unclear, with the alternatives being either some natural disease process or covert abuse by a psychologically disturbed parent. Before the appropriate remedial course of action can be determined this diagnostic uncertainty must be remedied. Separation of child and parent may afford one method. If the disorder persists then clearly it cannot be caused by the separated parent; but cessation of the disorder during separation affords only presumptive evidence that the suspected parent was responsible. Such evidence is often both morally and legally insufficient to justify separation of parent and child until psychological treatment has been both accepted and deemed to have succeeded. By contrast covert surveillance of carefully selected children and their suspected parents seems rapidly to provide, in a remarkably high proportion of cases, visually recorded and at least legally acceptable proof of the suspected abuse.

Such is the dilemma, and neither approach to resolving it is morally comfortable precisely because each overrides some important moral concern. Nonetheless protection of children from lifethreatening but covert abuse from their parents in cases where there is a strong likelihood of such abuse seems likely to be widely agreed to take moral priority over the moral obligation not to deceive, and not to infringe upon the privacy of, the suspected abuser, even though there is a risk that innocent parents will as a result from time to time be wronged. If this assumption is correct then covert video surveillance will be widely agreed to be a justified, though morally disturbing, technique of investigation in some cases of suspected child abuse.

Even if so, we surely need wider public debate about morally acceptable criteria for the use of such covert surveillance in hospitals and about the appropriate controls needed to prevent abuse of the method and reassure the community that such surveillance is used only where no available alternative can be expected to protect the child better. It seems reasonable to propose that among such controls should be included both consideration by an ethics committee (whether research ethics committee or clinical practice ethics committee seems less important); and approval of individual cases by a judge or magistrate. But as with all serious moral dilemmas, no matter which way we resolve them we will be left with a sense of profound moral unease.

\section{References}

(1) Evans D. The investigation of life-threatening child abuse and Munchausen syndrome by proxy. Fournal of medical ethics 1995; 21: 9-13.

(2) Southall D, Samuels M. Some ethical issues surrounding covert video surveillance - a response. fournal of medical ethics 1995; 21: 104-105, 115. 